\title{
TEORES ELEVADOS DE COBRE E NÍQUEL EM DOIS SOLOS CULTIVADOS COM LARANJA NO ESTADO DE SÃO PAULO
}

\author{
Lidia Maria de Almeida Plicas ${ }^{(1)}$
}

Bianca Villas Bôas da Silva ${ }^{(2)}$

Flávio Fernando Manzini ${ }^{(3)}$

Resumo: O cultivo de citrus é uma das principais atividades agrícolas no Estado de São Paulo, sendo realizado em solos gerados a partir de diferentes materiais que conferem, naturalmente, variações na constituição química e mineralógica destes, além de poderem, ou não estar contaminados por metais tóxicos oriundos dos métodos defensivos. Com o objetivo de quantificar os atributos químicos e os teores de metais pesados do solo foi realizado um estudo comparativo entre solos assim cultivados, das regiões das cidades de Mogi Guaçu e São José do Rio Preto. Este estudo envolveu a determinação do pH do solo, análises volumétricas para quantificação dos teores de matéria orgânica, $\mathrm{Ca}^{+2}, \mathrm{Mg}^{+2}$ e $\mathrm{Al}^{+3}$, espectrofotômetro de chama para o estabelecimento dos teores de $\mathrm{Na}^{+}$e $\mathrm{K}^{+}$e espectrofotômetro de absorção atômica para determinação dos teores de Cobre, Zinco, Cádmio, Cromo, Níquel e Chumbo. O solo da região de Mogi Guaçu apresentou-se mais ácido devido, provavelmente, a uma maior concentração de $\mathrm{Al}^{3+}$. Apresentou, também, teores mais elevados de matéria orgânica. Os solos de ambas as regiões apresentaramse deficientes em $\mathrm{Na}^{+}, \mathrm{K}^{+}, \mathrm{Ca}^{+2}$ e $\mathrm{Mg}^{+2}$. A análise realizada para os metais pesados mostrou uma elevada quantidade de Níquel observada nas amostras de solo da região de

\footnotetext{
${ }^{1}$ Professora Assistente Doutora em Química do Departamento de Química e Ciências Ambientais, Instituto de Biociências, Letras e Ciências Exatas - IBILCE - UNESP. São José do Rio Preto (SP). e-mail: plicas@ibilce.unesp.br

${ }^{2}$ Bacharel em Química Ambiental. Instituto de Biociências, Letras e Ciências Exatas - IBILCE - UNESP. São José do Rio Preto (SP). e-mail: bibarion@hotmail.com

${ }^{3}$ Professor Assistente Doutor em Geologia Regional do Departamento de Química e Ciências Ambientais, Instituto de Biociências, Letras e Ciências Exatas - IBILCE - UNESP. São José do Rio Preto (SP). e-mail: fmanzini@ibilce.unesp.br
} 
São José do Rio Preto e, Níquel e Cobre, nas de Mogi Guaçu, com valores classificados como de prevenção, de acordo com os Parâmetros Orientadores da CETESB (2005). Estes teores de alerta devem-se, muito possivelmente, à adição exagerada de defensivos agrícolas.

Palavras-chave: Atributos químicos do solo. Metais pesados. Citrus.

\section{INTRODUÇÃO}

O Estado de São Paulo lidera, no cenário nacional, o cultivo de citrus, especialmente a produção de laranja, limão e tangerina que ocupam, em conjunto, quase 800.000 ha de área plantada no estado. Destaca-se a região de Mogi Guaçu, com mais de 17.500 ha de área plantada com laranja, responsável por 2,37\% da produção estadual da fruta de acordo com o PROJETO LUPA (2007/2008) que corresponde ao censo agropecuário realizado pela Secretaria de Agricultura e Abastecimento do Estado de São Paulo (SAA) e pela Coordenadoria de Assistência Técnica Integral/Instituto de Economia Agrícola (CATI/IEA).

Para manter esta alta produtividade por vezes ocorrem descuidos no manejo do solo, podendo transformá-lo em importante fonte de contaminação. A utilização, de maneira não apropriada, de defensivos agrícolas, de águas de irrigação de baixa qualidade e a disposição indiscriminada de resíduos industriais ou domésticos pode provocar, por exemplo, o acúmulo de substâncias impróprias, em especial, metais pesados, que podem ser tóxicas para plantas podendo entrar na cadeia alimentar, afetando o ser humano (Guaracho \& Ponte, 2005).

Metais pesados são elementos de alta densidade, em comparação a outros elementos comuns. Apresentam densidade igual ou superior a $5 \mathrm{~g} / \mathrm{cm}^{3}$, e número atômico maior que 20 (Soares, 2004). Essa expressão "metais pesados", de definição muito criticada e controvertida, é utilizada na literatura relacionada a metais, semi-metais e nãometais. São utilizados como sinônimos, "metais traço", "elementos traço", "micronutrientes", "microelementos", entre outros. De qualquer forma, todo o conjunto 
de metais pesados, presente no solo, deve ser monitorado, pois apesar de alguns serem essenciais ao desenvolvimento de vida, tornam-se tóxicos a partir de determinadas concentrações constituindo risco aos participantes de toda a cadeia alimentar. Exceção seja feita ao cádmio, chumbo e mercúrio pelo fato de serem carcinogênicos, mesmo em pequenas concentrações. Com relação à toxicidade do níquel, há uma série de relatos classificando-o como carcinógeno às vias respiratórias quando inalado, porém poucos estudos existem sobre os efeitos de seu acúmulo no organismo (Duarte \& Pasqual, 2000). Quanto ao cobre, Casali et. al. (2008) reportam que as adições freqüentes deste metal no solo mediante sucessivas aplicações de fungicidas cúpricos podem aumentar a quantidade de formas solúveis, potencializando a toxidez às plantas e sua transferência, via sedimentos, para mananciais de águas superficiais. No homem, seu excesso pode provocar degeneração hepática e distúrbios neurológicos, entre outros (Brito et al., 2005). $\mathrm{O}$ destaque dado aos dois metais acima se justifica porque foram detectados teores importantes dos mesmos durante a realização dos estudos.

O objetivo deste trabalho foi a caracterização dos atributos químicos e a determinação dos teores de metais pesados em amostras de solos cultivados com laranja das regiões das cidades de São José do Rio Preto e Mogi Guaçu, no Estado de São Paulo.

\section{DESENVOLVIMENTO}

\subsection{Amostragem}

Foram coletadas amostras da camada superficial $(0$ a $20 \mathrm{~cm})$, de acordo com os procedimentos descritos em Silva (1999), de dois solos do Estado de São Paulo: ARGISSOLO VERMELHO-AMARELO Eutrófico (Oliveira, 1999), da região de São José do Rio Preto, parental das rochas sedimentares fluviais da Formação São José do Rio Preto, unidade litoestratigráfica do topo da pilha sedimentar do Grupo Bauru, neocretáceo da Bacia Bauru (Fernandes \& Coimbra, 2000), e LATOSSOLO VERMELHO-AMARELO Distrófico A (Oliveira, 1999), da região de Mogi Guaçu, parental das formações sedimentares glaciais do Grupo Tubarão de idade permo-carbonífero (IPT, 1981). Estas 
amostras foram submetidas à secagem ao ar e passadas por peneira com 2,00 mm de malha (terra fina seca ao ar - TFSA).

\subsection{Composição granulométrica}

A granulometria do solo foi estabelecida utilizando-se a metodologia de pipetagem proposta por Suguio (1973).

\subsection{Análises químicas}

As amostras coletadas foram submetidas a métodos de análises químicas para determinação do seu grau de acidez $(\mathrm{pH})$, da quantidade de matéria orgânica, dos teores de $\mathrm{Na}^{+}, \mathrm{K}^{+}, \mathrm{Ca}^{+2}, \mathrm{Mg}^{+2}$ e $\mathrm{Al}^{+3}$ e dos teores de cobre, zinco, cádmio, cromo, níquel e chumbo.

$\mathrm{O} \mathrm{pH}$ das amostras de solo foi determinado adicionando-se uma quantidade de 10 $\mathrm{cm}^{3}$ de TFSA a $25 \mathrm{~mL}$ de solução de $\mathrm{CaCl}_{2}$ 0,01 mol. $\mathrm{L}^{-1}$. As amostras foram agitadas e deixadas em repouso por 15 minutos. Foram então, submetidas a mais 5 minutos de agitação e mais 30 minutos de repouso findos os quais se procedeu à leitura dos resultados (Silva, 1999).

Para a obtenção do teor de matéria orgânica dos solos, foi utilizada a metodologia volumétrica descrita por Camargo et al. (1986) na qual se procede à oxidação da matéria orgânica do solo com solução de dicromato de potássio em presença de ácido sulfúrico concentrado, utilizando-se como catalisador da oxirredução o calor desprendido na diluição do ácido sulfúrico e titulação do excesso de dicromato com sulfato ferroso amoniacal.

A extração de $\mathrm{Na}^{+}$e $\mathrm{K}^{+}$foi realizada com solução duplo ácida $\left(\mathrm{HCl}\right.$ 0,05 mol. $\mathrm{L}^{-1}+$ $\mathrm{H}_{2} \mathrm{SO}_{4}$ 0,0125 mol. $\mathrm{L}^{-1}$ ), utilizando-se de fotômetro de chama. Para o preparo da solução extratora e das soluções padrão de $\mathrm{Na}^{+}$(5, 10, 15 e 20 ppm) e K+ (20, 50, 70 e 100 ppm) foi aplicada a metodologia descrita por Silva (1999). A determinação dos teores dos elementos baseou-se na técnica postulada por Raij et al. (2001), na qual foram adicionados $50 \mathrm{~mL}$ de solução extratora duplo-ácida a $5 \mathrm{~cm}^{3}$ de solo contidos em frascos 
plásticos. Estes frascos foram tampados e agitados por 5 minutos a $220 \mathrm{rpm}$ e deixados para decantação por cerca de12 horas. Após este período, a solução foi filtrada e o extrato foi submetido à leitura no fotômetro para a quantificação dos íons de interesse.

A determinação de $\mathrm{Ca}^{+2}, \mathrm{Mg}^{+2}$ e $\mathrm{Al}^{+3}$ foi feita por método volumétrico a partir da extração com KCl, de acordo com metodologia adaptada de Silva (1999). Nesta análise foram utilizadas as soluções de EDTA $0,025 \mathrm{~mol}^{-L^{-1}}, \mathrm{KCl} 1,0 \mathrm{~mol} . \mathrm{L}^{-1}, \mathrm{NaOH} \mathrm{0,5} \mathrm{mol} . \mathrm{L}^{-1}$, $\mathrm{NaOH} 0,025$ mol.L-1 ${ }^{-1} \mathrm{NH}_{4} \mathrm{OH} 0,1$ mol...-1 (solução tampão $=\mathrm{pH} 10$ ), e os indicadores eriocromo $\mathrm{T}$, murexida e azul-de-bromotimol. Para a extração dos elementos foram acrescentados $100 \mathrm{~mL}$ da solução extratora $\mathrm{KCl}$ a $10 \mathrm{~cm}^{3}$ de solo. $\mathrm{O}$ sistema foi agitado por 5 minutos e deixado em repouso por 12 horas.

Para determinação do teor da associação $\mathrm{Ca}^{+2}+\mathrm{Mg}^{+2}$ foram pipetados $25 \mathrm{~mL}$ do sobrenadante anterior, adicionados $5,0 \mathrm{~mL}$ de $\mathrm{NH}_{4} \mathrm{OH}$ e $0,1 \mathrm{~g}$ de eriocromo T. A titulação foi processada com EDTA até a viragem para azul.

Para determinação do teor de $\mathrm{Ca}^{+2}$ foram pipetados $25 \mathrm{~mL}$ do mesmo sobrenadante, adicionado $1,0 \mathrm{~mL}$ de $\mathrm{NaOH}$ e $0,1 \mathrm{~g}$ de murexida. A titulação foi processada com solução de EDTA até a viragem para lilás.

O teor de $\mathrm{Mg}^{+2}$ foi determinado pela diferença do teor total menos o ter de $\mathrm{Ca}^{+2}$.

Para a determinação do teor de $\mathrm{Al}^{+3}$ foram pipetados $25 \mathrm{ml}$ do sobrenadante $\mathrm{e}$ adicionadas 3 gotas do indicador azul-de-bromotimol. A titulação foi processada com solução de $\mathrm{NaOH}$ 0,025 mol. $\mathrm{L}^{-1}$ até a viragem para verde.

A determinação quantitativa dos metais cobre, zinco, cádmio, cromo, níquel e chumbo nas amostras de solo foi realizada mediante a utilização de espectrofotômetro de absorção atômica de acordo com a metodologia encontrada em Raij et al. (2001). Para a obtenção das curvas de calibração foram utilizadas soluções padrão estoque (SpecSol) de concentração de $1.000 \mathrm{mg} \cdot \mathrm{L}^{-1}$ para cada metal, nas diluições necessárias. Para a determinação dos teores dos metais foram adicionados $40 \mathrm{~mL}$ de solução extratora de DTPA a $20 \mathrm{~cm}^{3}$ de solo contido em frasco plástico. Este frasco foi tampado e agitado por período de 2 horas a $220 \mathrm{rpm}$. A suspensão foi filtrada e submetida à análise no aparelho.

\subsection{Resultados e discussão}


Quanto à textura dos solos, proporcionada pelas análises granulométricas, os resultados indicaram que as amostras provenientes da região de São José do Rio Preto são, predominantemente, arenosas enquanto que as de Mogi Guaçu apresentaram um melhor equilíbrio entre as frações arenosa e fina (silte + argila) (Tabela 1).

Tabela 1. Granulometria das amostras de solo

\begin{tabular}{cccc}
\hline Amostras $^{(1)}$ & Argila (\%) & Silte (\%) & Areia (\%) \\
\hline $\mathbf{1}$ & 6,9 & 11,8 & 81,3 \\
$\mathbf{2}$ & 5,7 & 8,2 & 86,1 \\
$\mathbf{3}$ & 3,3 & 7,7 & 89,0 \\
$\mathbf{4}$ & 4,6 & 5,7 & 89,7 \\
$\mathbf{5}$ & 6,1 & 6,9 & 87,0 \\
Amostras & $(2)$ & & \\
$\mathbf{1}$ & 10,5 & 27,4 & 61,1 \\
$\mathbf{2}$ & 13,6 & 36,6 & 49,8 \\
$\mathbf{3}$ & 11,7 & 35,4 & 52,9 \\
$\mathbf{4}$ & 12,1 & 30,4 & 57,5 \\
$\mathbf{5}$ & 12,2 & 34,4 & 53,4 \\
\hline (1) $^{(1)}$ Amostras de solo da região de São José do Rio Preto. & ${ }^{(2)}$ Amostras de solo da região de Mogi Guaçu.
\end{tabular}

Os atributos químicos dos solos, $\mathrm{pH}$, teor de matéria orgânica e os teores dos elementos cálcio, magnésio, alumínio, sódio e potássio foram classificados de acordo com Tomé Júnior (1997) (Tabela 2), e foram utilizados os valores orientadores propostos pela CETESB (2005) para os teores dos metais cobre, zinco, cádmio, cromo, níquel e chumbo.

Tabela 2. Atributos químicos de um Argissolo Vermelho-Amarelo e um Latossolo Vermelho-Amarelo cultivados com laranja 


\section{FÓRUM AMBIENTAL DA ALTA PAULISTA}

Volume VI - Ano 2010

Instituição Organizadora: ANAP - Associação Amigos da Natureza da Alta Paulista

\begin{tabular}{|c|c|c|c|c|c|c|c|}
\hline Atributo & $\begin{array}{l}\mathrm{pH} \text { em } \\
\mathrm{CaCl}_{2}\end{array}$ & $\begin{array}{l}\text { M.O. } \\
\text { g kg }^{-1}\end{array}$ & $\begin{array}{c}\mathrm{Na}^{+} \\
(\mathrm{mmol} \\
\left.\mathrm{dm}^{-3}\right)\end{array}$ & $\begin{array}{c}\mathrm{K}^{+} \\
(\mathrm{mmol} \\
\left.\mathrm{dm}^{-3}\right)\end{array}$ & $\begin{array}{c}\mathrm{Ca}^{+2} \\
(\mathrm{mmol} \\
\left.\mathrm{dm}^{-3}\right)\end{array}$ & $\begin{array}{c}\mathrm{Mg}^{+2} \\
(\mathrm{mmol} \\
\left.\mathrm{dm}^{-3}\right)\end{array}$ & $\begin{array}{c}\mathrm{Al}^{+3} \\
(\mathrm{mmol} \\
\left.\mathrm{dm}^{-3}\right)\end{array}$ \\
\hline \multicolumn{8}{|c|}{ Amostras $^{(1)}$} \\
\hline 1 & 6,84 & 7,8 & 0,025 & 0,263 & 2,15 & 0,263 & 0,5 \\
\hline 2 & 6,50 & 11,9 & 0,016 & 0,229 & 1,25 & 0,229 & 1,25 \\
\hline 3 & 6,71 & 7,1 & 0,019 & 0,162 & 1,37 & 0,162 & 0,0 \\
\hline 4 & 6,34 & 5,3 & 0,016 & 0,197 & 1,15 & 0,197 & 1,0 \\
\hline 5 & 6,32 & 8,1 & 0,007 & 0,208 & 0,85 & 0,208 & 1,0 \\
\hline
\end{tabular}

Amostras $^{(2)}$

\begin{tabular}{|c|c|c|c|c|c|c|c|}
\hline 1 & 4,68 & 16,9 & 0,021 & 0,262 & 0,85 & 0,57 & 3,75 \\
\hline 2 & 5,83 & 20,4 & 0,027 & 0,265 & 2,37 & 0,64 & 2,0 \\
\hline 3 & 5,30 & 10,8 & 0,023 & 0,213 & 1,55 & 0,60 & 1,0 \\
\hline 4 & 5,63 & 16,9 & 0,023 & 0,228 & 1,82 & 0,57 & 1,0 \\
\hline 5 & 5,80 & 14,1 & 0,019 & 0,253 & 1,87 & 0,40 & 1,0 \\
\hline
\end{tabular}

As amostras de solo da região de São José do Rio Preto apresentaram acidez muito baixa o que significa que podem apresentar altos teores de $\mathrm{Ca}^{+2}, \mathrm{Mg}^{+2} \mathrm{e} \mathrm{K}^{+}$, com deficiência de micronutrientes. O solo amostrado da região de Mogi Guaçu apresentou certa heterogeneidade, com amostras de acidez alta, amostras de acidez média e uma amostra com acidez baixa.

Com relação ao teor de matéria orgânica do solo (MOS) as amostras provenientes de São José do Rio Preto apresentaram baixos teores, provavelmente, correlacionados à constituição dos solos, que por serem muito arenosos e altamente porosos, são passíveis de rápida percolação por águas o que facilita a lixiviação de bases e os empobrece, não somente em matéria orgânica, mas também, nos demais nutrientes. Já as amostras de 
solo provenientes de Mogi Guaçu apresentaram, preferencialmente, médios teores de MOS relacionados, com certeza, ao maior equilíbrio na constituição do solo, menos arenoso e com maior contribuição da fração fina silto-argilosa.

Segundo Tomé Júnior (1997), para os solos do Estado de São Paulo, o valor menor ou igual a 1,0 mmol.dm ${ }^{-3}$ é considerado baixo teor para o íon potássio. Os solos de São José do Rio Preto e de Mogi Guaçu apresentaram valores de $\mathrm{K}^{+}$inferiores ao parâmetro acima, portanto, deficientes do mesmo. As concentrações de $\mathrm{Na}^{+}$nas amostras analisadas são ainda menores do que as determinadas para $\mathrm{K}^{+}$, como o esperado para solos não salinos, não havendo a possibilidade de prejuízo à cultura.

Solos com concentrações de $\mathrm{Ca}^{+2}$ menores que 20,0 mmol. $\mathrm{dm}^{-3}$ e de $\mathrm{Mg}^{+2}$ menores que 4,0 mmol.dm ${ }^{-3}$ para o Estado de São Paulo são considerados de baixo teor disponível destes elementos. Todas as amostras analisadas apresentaram valores inferiores aos limites citados. As concentrações dos íons $\mathrm{Ca}^{+2}$ e $\mathrm{Mg}^{+2}$ estão, diretamente, relacionadas ao nível de acidez do solo, ou seja, quanto maiores estas concentrações maior será o pH, portanto mais alcalino o solo (Tomé Júnior, 1997). Este fato está demonstrado nas análises realizadas onde, dentre as amostras de solo de São José do Rio Preto, a amostra 5 apresentou o menor valor de $\mathrm{pH}$ e a menor concentração de $\mathrm{Ca}^{+2}$. Quanto às amostras de solos de Mogi Guaçu, a amostra 1, com o menor valor de $\mathrm{pH}$ do conjunto, também foi a que mostrou maior deficiência em $\mathrm{Ca}^{+2}$.

$\mathrm{O} \mathrm{Al}^{+3}$ é um íon tóxico para as plantas sendo ideal que sua concentração seja nula no solo, porém, concentrações de até $5,0 \mathrm{mmol}^{-\mathrm{dm}^{-3}}$ são consideradas aceitáveis (Tomé Júnior, 1997). Os solos estudados apresentaram concentrações baixas em $\mathrm{Al}^{+3}$, destacando-se o resultado obtido para a amostra 1 do solo de Mogi Guaçu, cujo teor mais elevado é condizente com o menor valor de $\mathrm{pH}$.

Para a detecção dos teores dos metais cobre, zinco, cádmio, cromo, níquel e chumbo foi, inicialmente, necessária a obtenção dos valores de limite de detecção para cada elemento (Tabela 3).

Tabela 3: Valores de Limite de Detecção (L.D.) para os elementos analisados

\begin{tabular}{|c|c|c|c|c|c|c|}
\hline Elementos & $\mathbf{C u}$ & $\mathbf{Z n}$ & $\mathbf{C d}$ & $\mathbf{C r}$ & $\mathbf{N i}$ & $\mathbf{P b}$ \\
\hline L.D. (mg.kg-1) & 0,003 & 0,004 & 0,029 & 0,017 & 0,039 & 0,094 \\
\hline
\end{tabular}


Após a determinação das absorbâncias dos referidos elementos e realizadas as correções com o uso das curvas de calibração foram obtidas as suas concentrações (Tabela 4).

Tabela 4. Concentrações de metais das amostras de solo de São José do Rio Preto e de Mogi Guaçu

\begin{tabular}{|c|c|c|c|c|c|c|}
\hline Amostras $^{(1)}$ & $\begin{array}{c}\mathrm{Cu} \\
\left(\mathrm{mg} \cdot \mathrm{kg}^{-1}\right)\end{array}$ & $\begin{array}{c}\mathrm{Zn} \\
\left(\mathrm{mg}^{\prime} \mathrm{kg}^{-1}\right)\end{array}$ & 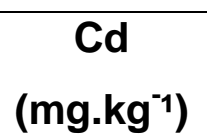 & $\begin{array}{c}\mathrm{Cr} \\
\left(\mathrm{mg} \cdot \mathrm{kg}^{-1}\right)\end{array}$ & $\begin{array}{c}\mathrm{Ni} \\
\left(\mathrm{mg}^{\prime} \mathrm{kg}^{-1}\right)\end{array}$ & $\begin{array}{c}\mathrm{Pb} \\
\left(\mathrm{mg} \cdot \mathrm{kg}^{-1}\right)\end{array}$ \\
\hline 1 & 32,7 & 5,4 & $<$ L.D $^{(3)}$ & $<$ L.D & 66,6 & $<$ L.D \\
\hline 2 & 10,8 & 5,2 & $<$ L.D & $<$ L.D & 67,4 & $<$ L.D \\
\hline 3 & 7,5 & 4,7 & $<$ L.D & $<$ L.D & 66,1 & $<$ L.D \\
\hline 4 & 4,7 & 3,9 & $<$ L.D & $<$ L.D & 68,3 & $<$ L.D \\
\hline 5 & 7,2 & 8,1 & $<$ L.D & $<$ L.D & 66,9 & $<$ L.D \\
\hline
\end{tabular}

\section{Amostras $^{(2)}$}

$\begin{array}{lllllll}\mathbf{1} & 56,5 & 20,5 & \text { < L.D } & <\text { L.D } & 66,6 & <\text { L.D } \\ \mathbf{2} & 77,3 & 20,4 & \text { < L.D } & <\text { L.D } & 65,7 & <\text { L.D } \\ \mathbf{3} & 100,3 & 7,3 & \text { < L.D } & <\text { L.D } & 65,2 & <\text { L.D } \\ \mathbf{4} & 47,9 & 8,8 & \text { < L.D } & <\text { L.D } & 65,0 & <\text { L.D } \\ \mathbf{5} & 71,7 & 9,7 & \text { < L.D } & <\text { L.D } & 65,0 & <\text { L.D }\end{array}$

${ }^{(1)}$ Amostras de solo da região de São José do Rio Preto. ${ }^{(2)}$ Amostras de solo da região de Mogi Guaçu. ${ }^{(3)}$ Limite de detecção.

A contaminação do solo por elementos tóxicos é uma forma de degradação ambiental devida, principalmente, à crescente contribuição antrópica, afetando sua qualidade e capacidade produtiva. Portanto concentrações excessivas de metais essenciais e não essenciais resultam em fitotoxicidade. Cádmio e chumbo não apresentam função biológica para as plantas. Zinco, cobre, níquel e cromo são essências 
às plantas, animais e microorganismos em pequenas quantidades. São potencialmente tóxicos em concentrações elevadas (Siqueira et al.,1994).

Segundo Silva et al. (2008), o aumento da concentração e da mobilização de metais nos solos se deve, em grande parte, ao uso de fertilizantes fosfatados. A presença no ambiente de altas concentrações destes metais pode causar vários problemas, pois estes se bioacumulam (biomagnificação) na cadeia alimentar, acarretando distúrbios nos processos metabólicos dos seres vivos.

Nas análises realizadas, os elementos cádmio, cromo e chumbo, apresentaram teores abaixo do limite de detecção. Este fato, porém, não significa, necessariamente, a não existência dos mesmos nas amostras, uma vez que a metodologia utilizada no presente trabalho, objetivou a determinação dos teores disponíveis e não totais destes metais.

Quanto ao zinco, é um elemento essencial aos homens, animais e plantas. Em condições tropicais, seu teor no solo oscila entre 10 e $30 \mathrm{mg}^{\mathrm{kg}}{ }^{-1}$. Esta faixa é considerada de baixo teor. Excesso de zinco no solo raramente é detectado, exceto próximo a depósitos, minas ou indústrias de chumbo e zinco (Macêdo, 2008). Todas as amostras analisadas apresentaram teores do elemento dentro da faixa de referência de qualidade da CETESB (2005).

Os estudos realizados para a obtenção dos teores de cobre revelaram que as amostras de solo de São José do Rio Preto possuem concentração do elemento dentro dos níveis aceitáveis. As amostras de solo provenientes de Mogi Guaçu apresentaram teores classificados como de prevenção de acordo com os parâmetros utilizados como referência. A contaminação dos solos por compostos contendo cobre resulta da utilização de materiais tais como spray (pulverizado como defensivo), fertilizantes, resíduos agrícolas e urbanos, assim como, de emissões industriais (Kabata-Pendias \& Pendias, 1984). Conforme Guilherme et al. (2005), algumas das contribuições de insumos agrícolas na contaminação de solos com cobre são: fertilizantes fosfatados de 1 a $3000 \mathrm{mg} \cdot \mathrm{kg}^{-1}$, calcários de 2 a $125 \mathrm{mg} \cdot \mathrm{kg}^{-1}$, fertilizantes nitrogenados de 1 a $15 \mathrm{mg} \cdot \mathrm{kg}^{-1}$ e agrotóxicos de 4 a $50 \%$.

As análises realizadas para os teores de níquel das amostras de solo para ambas as regiões revelaram teores próximos aos valores de intervenção sendo necessário um 
maior monitoramento com relação a este elemento. A aplicação de iodo e certos fertilizantes fosfatados pode ser importante fonte poluidora deste metal (Kabata-Pendias \& Pendias, 1984) e deve ser a provável razão dos teores elevados detectados.

\section{CONCLUSÃO}

Dentre todas as análises realizadas para as amostras de solos, cultivados com laranja, das regiões de São José do Rio Preto e Mogi Guaçu, destacam-se os valores relativamente elevados, obtidos para níquel e cobre. Levando-se em consideração que os teores destes metais foram determinados utilizando-se DTPA, que é um extrator para quantidades biodisponíveis dos mesmos, os valores são, portanto, preocupantes uma vez que, se fosse empregada a metodologia para a determinação dos teores totais, muito provavelmente, seriam detectados valores ainda mais elevados. Apesar destes valores enquadrarem-se dentre aqueles considerados de prevenção pela CETESB, estes solos não necessitam de remediação desde que sejam mantidos sob monitoramento constante visando prevenir os riscos de contaminação e poluição envolvidos.

\section{REFERÊNCIAS}

BRITO, J.C.F.; COUTINHO, M.A.P.; ALMEIDA, H.J.F. \& NÓBREGA, P.V. Doença de Wilson diagnóstico clínico e sinais das "Faces do Panda" à ressonância magnética. Relato de caso. Arq Neuropsiquiatr, 63:176-179, 2005.

CAMARGO, O.A.; MONIZ, A.C.; JORGE, J.A. \& VALADARES, J.M.A.S.- Métodos de análise química, mineralógica e física de solos do Instituto Agronômico de Campinas - Boletim Técnico do Instituto Agronômico, Campinas, 1986, 94p.

CASALI, C.A.; MOTERLE, D.F.; RHEINHEIMER, D.S.; BRUNETTO, G.; CORCINI, A.L.M.; KAMINSKI, J. \& MELO, G.W.B. Formas e dessorção de cobre em solos cultivados com videira na Serra Gaúcha do Rio Grande do Sul. R. Bras. Ci. Solo, 32:1479-1487, 2008. 
COMPANHIA DE TECNOLOGIA DE SANEAMENTO AMBIENTAL - CETESB. Valores orientadores para solos e águas subterrâneas no Estado de São Paulo. Disponível em: <http: // www.agsolve.com.br/pdf/artigos/novatabelacetesb2005.pdf> Acesso em: 9 nov. de 2009.

DUARTE, R.P.S. \& PASQUAL, A. Avaliação do cádmio (Cd), Chumbo (Pb), Níquel (Ni) e Zinco (Zn) em solos, plantas e cabelos humanos. Energia na Agricultura, 15:46-58, 2000.

FERNANDES, L.A. \& COIMBRA, A.M. Revisão estratigráfica da parte oriental da Bacia Bauru (Neocretáceo). R. Bras. Geociênc., 30:717-728, 2000.

GUARACHO, V.V. \& PONTE, M.J.J.S. Utilização da Técnica de Remediação Eletrocinética para Remoção de Chumbo e Níquel de Landfarming de Refinaria de Petróleo. In: CONGRESSO P\&D EM PETRÓLEO E GÁS, 3., 2005. Anais. Salvador, 2005. CD-ROM.

GUILHERME, L.R.G.; MARQUES, J.J.G.S.M.; PIERANGELI, M.A.P.; ZULIANI, D.Q. \& CAMPOS, M.L. Elementos-traço em solos, sedimentos e nas águas. Tópicos em ciência do solo, vol. 5. Sociedade Brasileira de Ciência do Solo, Viçosa, 2005. p.345390.

INSTITUTO DE PESQUISAS TECNOLÓGICAS DO ESTADO DE SÃO PAULO - IPT. Mapa geológico do Estado de São Paulo. Escala 1:500.000. Governo do Estado de São Paulo. Secretaria da Indústria, Comércio, Ciência e Tecnologia, 1981. 126p. (Monografias 6).

KABATA-PENDIAS, A. \& PENDIAS, H. Trace elements in soils and plants. Boca Raton, CRC Press, 1984. 315p. 
SILVA, Z.M.C.V.A; LACERDA, J.J.J.; FRANÇA, R.S.; SANTOS, J.S.; SANTOS. M.L.P.; Extração assistida por ultra-som para avaliação da disponibilização de metais pesados em solos agrícolas no Sudoeste da Bahia. In: ENCONTRO NACIONAL DE QUÍMICA AMBIENTAL, 4., Aracaju, 2008. Aracaju, 2008. CD-ROM.

MACÊDO, L.S. \& MORRIL, W.B.B. Origem e comportamento dos metais fitotóxicos: Revisão da literatura. Tecnol. \& Ciên. Agropec., 2:29-38, 2008.

OLIVEIRA, J.B. Solos do Estado de São Paulo: descrição das classes registradas no mapa pedológico. Campinas, Instituto Agronômico, 1999. 112p. (Boletim Científico 45).

PROJETO LUPA. SÃO PAULO (Estado). Secretaria de Agricultura e Abastecimento. Coordenadoria de Assistência Técnica Integral. Instituto de Economia Agrícola. Levantamento censitário de unidades de produção agrícola do Estado de São Paulo LUPA 2007/2008. São Paulo: SAA/CATI/IEA, 2008. Disponível em: <http://www.cati.sp.gov.br/projetolupa>. Acesso em: 16 out. de 2009.

RAIJ, B.; ANDRADE, J.C.; CANTARELLA, H. \& QUAGGIO, J.A., eds. Análise química para avaliação da fertilidade de solos tropicais. Campinas, Instituto Agronômico de Campinas, 2001, 285p.

SILVA, F.C. Manual de análises químicas de solos, plantas e fertilizantes. Embrapa Solos (Rio de Janeiro)/Embrapa Informática Agropecuária (Campinas), 1999, 370p.

SIQUEIRA, J.O.; MOREIRA, F.M.S; GRISI, B.M.; HUNGRIA, M. \& ARAÚJO, R.S. Microrganismos e processos biológicos do solo: perspectiva ambiental. Brasília, Embrapa, 1994. 142p. (Documento 45).

SOARES, M.R. Coeficiente de distribuição $(\mathrm{Kd})$ de metais pesados em solos do Estado de São Paulo. Piracicaba, Escola Superior de Agricultura de Piracicaba, Universidade de São Paulo, 2004. 202p. (Tese de Doutorado) 
SUGUIO, K. Introdução à sedimentologia. São Paulo, Ed. Edgard Blücher, 1973, 317p.

TOMÉ JÚNIOR, J.B. Manual para interpretação de análise de solo. Guaíba, Ed. Agropecuária, 1997, 247p. 